\title{
Fossil Magnetic Fields and Activity of Young Stars
}

\author{
A.E. Dudorov, E.E. Gorbenko \\ Astronomical Department of Moscow State University, \\ Sternberg State Astronomical Institute, Universitetskij Pr., 13, \\ 119899 Moscow V-234, USSR
}

\begin{abstract}
The strength of fossil magnetic fields in young stars is estimated. The interaction of a fossil magnetic field with convection in stellar envelopes is investigated. The influence of such a magnetic field, transformed to a small scale, on the activity of young stars is discussed.
\end{abstract}

1. The theory of the fossil magnetic field of stars has been developed recently with the help of numerical simulations of star formation in interstellar magnetic molecular clouds (see Dudorov, 1988, 1990). In the framework of this theory the magnetic field of protostellar clouds is transformed during star formation into the fossil magnetic field of young stars. The energy of the stellar fossil magnetic field is $10-10^{5}$ times lower than that of the corresponding frozen-in field. It depends on the efficiency of magnetic ambipolar and Ohmic diffusion, processes that occur in the protostellar cloud cores, with ionization ratio $x<10^{-12}$, and densities $n=\left(10^{4}-10^{10}\right) n_{o}$, where $n_{o}$ is the initial number density of the cloud.

Magnetic diffusion leads to the fragmentation of molecular cloud cores. In the process of magnetic fragmentation, magnetic protostars are formed with masses $M>(0.1-0.3) M_{\odot}$, with the initial ratio of magnetic energy to gravitational $e_{m}$ $=0.1-0.3$, and with the corresponding ratio for rotational energy, $e_{\omega}=0.01-0.1$. The oblate magnetic protostellar cloud continues to flatten during the accretion stage under the influence of electromagnetic and centrifugal forces and as the result of the action of magnetic torques and flow along the rotational axis.

In the protostars and in newborn stars that are still accreting mass, magnetic flux is pumped from the envelope of the protostellar cloud. After magnetosphere formation, the pumping of magnetic field from the accretion disk weakens. The interaction of the magnetosphere and accretion disk leads to outflows and jets along the lines of force of the magnetic field of the molecular cloud. In the boundary region between the magnetosphere and the inner edge of the accretion disk, the additional acceleration of charged particles along open magnetic lines may occur. The ejection of 30 per cent of the incoming mass flow from the disk is sufficient 
to maintain the angular velocity of such young stars (accretion stage of T Tauri stars) at about of $20-30 \mathrm{~km} / \mathrm{s}$.

2. Analyses of early stages of stellar evolution leads to the conclusion that efficient convection is absent from the envelopes of stars with masses above $2 M_{\odot}$. These stars can retain a strong fossil magnetic field in their envelopes. For this to happen, strong sources of ionization, SN or OB stars, must be present nearby. Intense cosmic rays, the depletion of dust or an excess of heavy elements in protostellar matter, all help to produce a fossil field with a surface intensity $B_{s} \geq 500-1000 \mathrm{G}$ (Dudorov and Tutukov, 1990).

The results of calculations show that the magnetic field strength inside cool active stars with masses $M<1.5-2.0 M_{\odot}$ in the T Tau stage is a hundred times lower than that of the frozen-in field. After the Hayashi stage the internal magnetic field may be of order $10^{6}-10^{7} \mathrm{G}$ at the core boundary and $10^{4}-10^{6} \mathrm{G}$ near the base of the convection zone (Dudorov, 1986; Dudorov et al., 1989). The surface fossil magnetic field of normal stars increases along the ZAMS from $B_{f s}=1-10 \mathrm{G}$ (M-stars) to $B_{f s}=100-200 \mathrm{G}$ (B-stars). The magnetic energy of these stars is $E_{m}=10^{-1}-10^{-2} E_{g}\left(E_{g}-\right.$ gravitational energy).

3. The subsequent evolution of the fossil magnetic field depends on hydromagnetic or resistive instabilities, buoyancy, Ohmic and turbulent diffusion. The turbulent convection in newborn stars on the Hayashi stage converts large scale magnetic field into a small scale, because the convective energy may be as large as the thermal. The buoyancy of magnetic flux tubes can cause the activity of the pre-main sequence stars. On the other hand turbulent convection together with the differential rotation enables the operation of an $\alpha \omega$-dynamo, which turns on after sufficient turbulent dissipation of fossil magnetic field. The differential rotation is generated by the gravitational compression of protostars (Dudorov et al., 1989).

4. The results of numerical calculations and estimates show that the strength of the fossil magnetic field inside the Sun in the T Tauri stage (Dudorov et al., 1989) exceeds the strength of the dynamo magnetic field by a factor of more than $10-$ 100. The difference of energies of these types of fields may appear as an energy of stellar activity which is a result of convective conversion of large-scale fossil field into turbulent magnetic field and a result of the evolution of small-scale magnetic field in convective and radiative stellar envelopes. The slender magnetic flux tubes, rising to stellar surface, cause the activity of young stars. Numerical experiments (Gorbenko and Dudorov, 1989) show that the basic contribution to the energetics of the activity of young stars is made by the largest existing magnetic flux tubes. This explains the attenuation of $T$ Tauri star activity with age.

In newborn stars magnetic flux tubes with the dimensions $d=0.05-0.1 R$ rise to the stellar surface ( $R$ the stellar radius). Their energies are $10^{30}-10^{32}$ erg.

In the post $T$ Tauri stars magnetic flux tubes with dimensions of order of pressure scale height are rising to the surface. The behaviour of the dynamo depends on the convective intensity and the differential rotation. The maximum effect is reached in G-stars. 
5. In K- and M-stars, magnetic ambipolar diffusion slows down the motion of slender magnetic flux tubes in the regions of partial hydrogen ionization (Dudorov, 1990).

In a newborn star, the magnetic diffusion zone is situated in the region of temperature minimum. A buoyancy mechanism may concentrate the magnetic flux tubes of medium dimensions beneath the temperature minimum, causing an increase of the radius of the star and also of its luminosity, giving rise to the mechanism of Gershberg (1983) as well as that hypothesised by Appenzeller and Dearborn (1984). In a such situation luminosity variations may arise, with amplitudes less than $2^{m}-3^{m}$.

\section{References}

Appenzeller, R.I., Dearborn, D.S.P.: 1984, Astrophys. J. 278, 689

Dudorov, A.E.: 1986, Astron. Tsirk., No 1446, 3

Dudorov, A.E.: 1989, Astron. Tsirk., No 1535, 25

Dudorov, A.E.: 1988, in Magnetic stars, eds. I.M.Kopylov, Yu.V.Glagolevskij, Nauka, Leningrad, p. 226

Dudorov, A.E.: 1990, Pis'ma Astron. Zh., in press.

Dudorov, A.E., Sazonov Yu.V.: 1987, Nauchn. Inf. 63, 68

Dudorov, A.E., Krivodubskij, V.N., Ruzmaikina, T.V., Ruzmaikin, A.A.: 1989, Astron. Zh. 68, No 4

Dudorov, A.E., Tutukov, A.V.: 1990, Astron. Zh. 67, 342

Gershberg, R.E.: 1983, Flare stars, Nauka, Moscow

Gorbenko, E.E., Dudorov, A.E.: 1989, Astron. Tsirk., No 1536, 27 